\title{
Physeal injuries in myelomeningocele patients
}

\author{
A Cuxart $\mathrm{MD},{ }^{1} \mathrm{~J}$ Iborra $\mathrm{MD},{ }^{2 *} \mathrm{M}$ Meléndez $\mathrm{MD},{ }^{3} \mathrm{E}$ Pagès $\mathrm{MD}^{3}$ \\ ${ }^{1}$ Senior staff, ${ }^{2}$ Junior staff, ${ }^{3}$ Resident, Rehabilitation Medicine Department, Spina Bifida \\ Unit, Hospital de Traumatologia y Rehabilitacion, C S Vall d'Hebron, Barcelona, Spain.
}

\begin{abstract}
Epiphysiolysis from a series of 253 patients under regular control in the Spina Bifida Unit at our hospital has been analysed from 1967 to the present time. From that total there have been 18 physeal fractures in 9 of the myelomeningocele patients. The clinical characteristics, diagnostic difficulties and therapeutic criteria for this type of lesion are considered, and we suggest the need to use suitable orthoses whenever the patient is able to walk, and to temporarily increase the level of orthotisation in patients if the physis of the knee is affected.
\end{abstract}

Keywords: physeal injuries; myelomeningocele; lower-extremity injuries.

\section{Introduction}

Painless fractures, often with no known history of trauma are relatively common in patients suffering from spina bifida sequelae. Fractures of the lower extremities occur in 10 to 30 percent of cases in patients with spina bifida. ${ }^{1}$ The fractures may involve the metaphysis, diaphysis, or physis. ${ }^{2,3}$ An association of spina bifida and epiphysiolysis, in contrast to what occurs with other fractures, is less frequent, less well known and may give rise to diagnostic error. Epiphysiolysis occurs in less than $10 \%$ of patients with spina bifida and myelomeningocele. ${ }^{4-7}$

The physis of the long bones of the lower limbs in myelomeningocele can be repeatedly injured during daily walking activities. In view of the lack of sensitivity in these patients it often happens that the fracture is only diagnosed some days after it has occurred, and it is often difficult to ascertain exactly when these fractures occurred. The diagnosis is commonly delayed, and this means that the healing process has already begun, ${ }^{8,9}$ and epiphyseal plate injuries present a truly alarming appearance in x-rays.

We review our experience with physeal injuries in the lower extremities in patients with myelomeningocele.

*Correspondence: Cami Ral No. 65 Esc. Izda. $3^{\circ} 2^{a}$, (08390) Montgat, Barcelona, Spain.

\section{Materials and methods}

A retrospective study was carried out on patients managed in our unit from 1967 to the present time, analysing a total of 253 patients. Eighteen physeal fractures were found in 9 of the patients. All the patients had myelomeningocele with variable neurological lesions (2 Th12, 1 L2, 5 L3, 1 L4).

At the time of the epiphysiolysis all those able to walk were orthetised. Six patients were walkers and 3 non walkers.

Both patients and/or their relatives were subjected to exhaustive questioning about the possible mechanisms of injury. A clinical and routine radiological study was carried out on all of the patients along with routine analytical control. Bone scans and CT scans were not carried out on any of the patients.

The 18 patients with epiphysiolysis were studied in accordance with the Salter \& Harris classification ${ }^{10}$ of epiphyseal plate injuries.

\section{Results}

Our patients consisted of 5 males and 4 females. The average age at the time of diagnosis of the lesion was 8.5 years $( \pm 3.7$, range 1-13).

Patients attended our unit at 9.2 days ( \pm 9.2 , range $0-30$ ) after the appearance of localised inflammatory signs (local warmth, erythema and swelling of the leg); in none 
was pain a relevant symptom, nor was there any fever. All of the patients who were able to walk usually did so in their own homes without any orthosis.

On clinical examination we found 3 patients with abnormal angulation or mobility on stressing. Only in 3 patients was there any known minor previous trauma. Regarding blood analysis, only one patient had a slight rise - the ESR (to 19), and slight leucocytosis (WBC 11,000).

The epiphysiolysis was localised as follows: 2 proximal femur, 5 distal femur, 2 proximal tibia and 9 distal tibia. Seven of these affected the right side and 11 the left.

In 5 cases the epiphysiolysis was localised at neurological level, that is in a sensitive area, and in 13 patients the localisation was below the neurological level, that is in an anaesthetic area.

The group of 6 walkers had 13 epiphysiolysis, and in the 3 non walkers, 5 had epiphysiolysis.

According to the Salter \& Harris classification the 18 examples of epiphysiolysis corresponded to types I (15) and II (3). Two $\mathrm{x}$-ray patterns appear; one with no epiphyseal displacement, corresponding to Salter \& Harris type I; and another type with displacement, type II, with $\mathrm{x}$-ray characteristics of physeal widening with irregular metaphysis that was dense and slightly widened due to subperiosteal haemorrhage. A periosteal reaction involving new bone formation was present.

Treatment was immobilisation in plaster (17) or surgery (one patient), depending on the type of physeal lesion.

The average length of treatment by immobilisation in plaster was 36.3 days ( \pm 18.2 , range $21-90)$.

Weight bearing began on withdrawal of immobilisation. The only patient presenting with displacement was treated on admission with reduction and temporary stabilisation with Kirschner wires, with immobilisation in plaster for 28 days.

Bone growth (Risser sign 5) had ended in 3 of the patients who had a total of 9 physeal lesions, and in another 3 patients growth was about to end (Risser sign 4), amounting to 6 physeal lesions; whilst in the remaining patients the Risser sign was 3 or less.
Nowadays the average age of such patients is 16.7 years $( \pm 2.7)$, with an average follow-up of 98.6 months $( \pm 65.5$, range 24-288).

Five physeal fractures showed complications, the most frequent being in the distal femur and the proximal tibia (4 out of 5). In 2 of the 3 patients with slight previous trauma some evidence of complications were observed. In all 5 cases fusion of the growth cartilage occurred causing up to $2 \mathrm{~cm}$ leg-length discrepancy with irregularities in the alignment of the affected limb, genu valgum, in 2 patients.

\section{Discussion}

In our unit $54 \%$ of myelomeningocele patients were male, the sex most frequently presenting epiphysiolysis, as is the case in healthy persons.

The peak incidence of physeal injury appears to be between the ages 5 and 13 years. Average patient age at the time the physeal injury occurred in over half of this series was around puberty, as is seen in healthy individuals. ${ }^{11}$

The presence of physeal fracture is usually discovered by the parents, who often first note increase in local warmth, swelling, gross deformity and crepitation. In none of the patients was pain a relevant symptom, nor was there fever.

It was not possible to establish the existence of serious trauma as the immediate cause of the condition in the majority of our patients, although on close examination of their history, all patients usually walked, or tried to walk without orthopaedic aid in their homes, which contributed to the production of lesions of this type due to chronic repeated stress over the physis. The bone most commonly involved is the tibia; next in frequency is the femur.

The most frequent localisation of epiphysiolysis is at the anaesthesic area, below the neurological level. The epiphysiolysis is more common in the flail epiphysis.

The said lesions may arise at any functional level, but are most frequent in the group of walking patients.

The radiological changes observed may 
give rise to diagnostic errors and unnecessary diagnostic attitudes, such as puncture biopsy or open biopsy. ${ }^{5,7,8,12,13}$ No biopsy of the epiphyseal plate was done on any of the patients in our series.

These are lesions behaving as in physeal processes with nonspecific inflammatory characteristics. ${ }^{13,14}$ The local nonspecific inflammation and metaphysepi-physary rarefaction is, we believe, due to a complex etiopathogenic sequence established when treating neuropathic joints which, due to a decrease in or absence of sensitivity, are more predisposed to developing pathological fractures brought about by chronic repeated stress, thus starting up a vicious circle consisting of chronic stress - lesion attempted repair - chronic stress - lesion. ${ }^{1,3,15}$

Therapy should be directed to treatment of the local condition with immediate immobilisation, avoiding weight bearing until a clinical cure is effected in lesser cases, and obligatory reduction in cases of most serious displacement (immobilisation for between 4-6 weeks). The basic principle for treatment of this type of pathological fracture is that the immobilisation time should be as short as possible. It is recommended that after an initial period of immobilisation and protection of the physis affected, the cast be removed and the limb supported in an orthosis allowing weight bearing and function. ${ }^{1.16}$

It is necessary to review the level of orthotisation in all cases of epiphysiolysis and in particular in those cases involving physes of the knee. Gyepes et al reported more physeal injuries in the region of the knee and the ankle in patients who do not use suitable orthotic support for walking. ${ }^{5}$ The level of orthotisation should be raised in cases where an insufficient quadriceps is foreseen, ie a muscular balance less than 3 (MRC scale) $)^{17}$ in order to decrease chronic stress on the knee physes.

It is of utmost importance to prevent serious sequelae such as growth arrest, stiffness of joints and alignment irregularities, ${ }^{18}$ which means routine examination of the weight bearing joints affected until growth ends.

\section{Conclusions}

1 Epiphysiolysis in these patients occurs with no previous serious trauma. Walkers are the patients with the most frequent incidence. Failure to use orthoses for walking in patients requiring these constitutes a risk factor with regard to epiphysiolysis; thus in view of the neurological characteristics of such patients it is necessary for them to walk with an orthopaedic aid at all times.

2 The condition arose in $61 \%$ of the patients in our series, at around puberty.

3 Although the characteristic clinical and radiological pattern together with analytical control were sufficient in all of our patients, CT scan and linear tomography are of great value in establishing an accurate diagnosis in the difficult cases.

4 Therapy is directed to treatment of the local condition with immediate immobilisation and avoiding any load until a clinical cure is obtained in lesser cases, and obligatory reduction in those with a serious displacement. In patients with physis of the knee and when the quadriceps strength is under 3 (MRC scale), the level of orthotisation should be increased temporarily. It means a change AFO by KAFO, in order to protect this joint from complications.

5 Physes in these patients should be controlled until growth ends.

\section{Dedication}

'A nos amis de l'Hôpital Lapeyronie, Montpellier.'

\section{References}

1 Menelaus MB (1980) The Orthopaedic Management of Spina Bifida Cystica. 2nd ed. E \& S Livingatone Ltd, Edinburgh: 63-65.

2 Korhonen BJ (1971) Fractures in Myelodisplasia. Clin Orthop 79: 145-150. 
3 Kumar SJ, Cowell HR, Townsend P (1984) Physeal metaphyseal and diaphyseal injuries of lower extremities in children. $J$ Pediatr Orthop 4: 25-27.

4 Soutter FE (1962) Spina bifida and epiphyseal displacement. J Bone Joint Surg 44B: 106-109.

5 Gyepes MT, Newbern DH, Neuhauser EBD (1965) Metaphyseal and physeal injuries in children with spina bifida and myelomeningocele. AJR 95: 168-177

6 Townsend PF, Cowell HR, Steg NL (1979) Lower extremity fractures simulating infection in myelomeningocele. Clin Orthop 144: 256-259.

7 Wenger DR, Jeffcoat BT, Herring JA (1980) The guarded prognosis of physeal injuries in paraplegic children. J Bone Joint Surg 62A: 241-246.

8 Navarro Quilis A (1974) Fractures in children with myelomeningocele. Acta Orthop Scand 45: 883-897.

9 Navarro A, Peiro A (1974) Healing in denervated bones. Acta Orthop Scand 45: 820-835

10 Salter RB Harris WR (1963) Injuries involving the epiphyseal plate. J Bone Joint Surg 45A: 587-622.

11 Alonso de Ros JF, Arcalis A, Villanueva C (1977) Desprendimientos epifisarios de la extremidad distal de la tibia. Revision de 62 casos. Rev Orthop Trauma 21 1B, no 3.

12 Peinado A (1973) Epifisiolisis y espina bifida. Cuadernos Clínicos 5: 24-26.

13 Cañadell J, de Pablos J (1988) Lesiones del Cartilago de Crecimiento. 2on. Ed Barcelona: Salvat.

14 Edvardsen P (1972) Physeo-epiphyseal injuries of lower extremities in myelomeningocele. Acta Orthop Scand 43: 550-557.

15 Johnson JTH (1967) Neuropathic fractures and joint injuries. Pathogenesis and rationale of prevention and treatment. J Bone Joint Surg 49A: 1-30.

16 Roberts JA, Bennet GC, MacKenzie JR (1989) Physeal widening in children with myelomeningocele. J Bone Joint Surg 71B: 30-32.

17 HMSO (1976) MRC grading memorandum No. 45.

18 Drabu KF, Walker G (1985) Stiffness after fractures around the knee in spina bifida. J Bone Joint Surg 67B: $266-267$. 